\title{
Generalization of the theory of critical distance to estimate lifetime of notched components in the medium-cycle fatigue regime
}

\author{
Rafael Borges, José Araújo, Cosme Silva, Jéssica Dias, Jorge Ferreira \\ University of Brasilia, Department of Mechanical Engineering, Brasilia, 70910-900, Brazil
}

\begin{abstract}
This paper proposes a new and improved way to determine the dependency of the parameter that characterizes the process zone size $l$ with fatigue life $N_{f}$. Thus, unlike the classical approach, this approach has the advantage of allowing the construction of a $l$ versus $N_{f}$ relationship, which is independent of the type of test used for calibration. In order to define the new relationship, a calibration strategy is presented considering the critical plane models based on the Fatemi-Socie and Smith-WatsonTopper. The accuracy of the method is verified by using data sets taken from the literature. A study on the effect of stress ratio (R) on the behaviour of the $l$ versus $N_{f}$ relationship is presented and indicates that, although such relationship seems to depend on the $\mathrm{R}$ value used in the calibration, the life predictions obtained by these relationships are statistically similar.
\end{abstract}

\section{Introduction}

This section briefly presents the critical distance theory $[1,2,3]$. The method aims to predict the fatigue strength of components with stress concentrators, such as notches or cracks [1]. In the Theory of Critical Distances, TCD, fatigue damage is predicted when the effective stress in a process zone exceeds the fatigue resistance of the material. Such effective stress can be found at a given point away from the hot spot (point method, PM), or by averaging along a line $(\mathrm{LM})$ or on an area (AM) or volume (VM) [1,2,3]. The critical distance, represented by the characteristic length $L$ varies for each method and can be calculated by Equation (1) when the point method is adopted.

$$
l=\frac{L}{2}=\frac{1}{2 \pi}\left(\frac{\Delta K_{t h}}{\sigma_{0}}\right)
$$

where $\sigma_{0}$ and $\Delta K_{t h}$ represent, respectively, the fatigue limit and the threshold value of the stress intensity factor range for long cracks. In the early 2000s, TCD was used to predict the multiaxial fatigue limit of notched components [4-15]. This intense investigation of TCD not only ensured an improvement in its formalization, but also made possible its extension to the finite life estimation under uniaxial conditions, by the definition of the critical distance versus life relationship, [4-8]. In the case of multiaxial fatigue analysis, TCD was coupled with the Modified Wohler Curve Method MWCM [9-13].

As an approximation, we can assume that TCD uses the maximum principal stress 
amplitude, $\sigma_{1_{a}}$, as a parameter associated with the driving force that induces fatigue failures. Although the idea of using such a stress parameter is quite convenient and simple, we must consider that in many situations it is not representative of the phenomenon. For example, this parameter discards the well known mean stress effect on fatigue resistance. In addition, depending on the material and stress state, the conditions of initiation and crack growth can be affected by the presence of normal and shear stress/strain components. Thus, from the phenomenological point of view, the use of $\sigma_{1_{a}}$ does not seem to be an adequate choice. In this sense, in order to solve this deficiency, some researchers have proposed critical distance models that use other parameters to represent the driving force that controls the fatigue phenomenon. Flavenot and Skalli [14], for example, defined a distance that depended on microstructural parameters called "critical depth" and used the shear and hydrostatic stress amplitudes to construct a criterion to determine the fatigue limit under multiaxial conditions. Castro et al. [15] used the criteria of Crossland, Dang Van and the MWCM to show that the critical distance may be different from half of the El Haddad's intrinsic crack length as proposed in [1].

\section{Calibration of critical versus life distance curve using parameters related to multiaxial models}

\subsection{Fatigue damage parameter}

The use of models based on critical plane approximations has become increasingly important due to the good results obtained in predicting life. The fundamental hypothesis of critical plane models assumes that the orientation of the microcracks can be identified by searching for the most damaged plane, among the possible crack initiation planes. The quantification of the level of fatigue damage introduced into a plane is expressed as a function of a parameter, called the damage parameter, which is usually represented as a function that combines the normal and shear stress components acting on that plane.

\subsubsection{Smith-Watson-Topper Parameter}

Based on the phenomenological hypothesis that the process of growth of microcracks in some materials follows planes of maximum strain or maximum normal stress, Socie [16] extended the use of the Smith-Watson-Topper model [17] to multiaxial fatigue. Considering this idea, it is possible to assume that, under elastic conditions, the critical plane will be the plane in which Equation (2) has a maximum value.

$$
P_{S W T}=\frac{\sigma_{a}}{E} \sigma_{n, \max }
$$

where $\sigma_{a}$ is the normal stress amplitude, $E$ is the modulus of elasticity, $\sigma_{n, \max }$ is the maximum normal stress on the maximum shear strain plane.

\subsubsection{Fatemi-Socie Parameter}

Fatemi-Socie model [18] was built inspired by Brown and Miller's work [19]. Under elastic conditions, the critical plane in this model can be defined as the material plane in which Equation (3) has a maximum value

$$
P_{F S}=\frac{\tau_{a}}{G}\left(1+k \frac{\sigma_{n, \max }}{s_{y}}\right)
$$


where $\tau_{a}$ is the shear stress amplitude, $k$ is a material constant $(0 \leq k \leq 1)$ and $S_{y}$ is the yield strength.

\subsection{Critical plane analysis considering the Fatemi-Socie and Smith-Watson- Topper models for plane problems and in-phase loading}

Under general loading conditions, the critical plane research can be performed using strategies and algorithms available in the literature [20,21]. However, for simpler situations, such as those observed in tests carried out under cyclic stress with non-zero mean stress, whose history is represented by Equation (4), it is possible to determine the SWT parameter by using Equation (5) and the FS parameter by considering Equations (6) and (7).

$$
\sigma(t)=\sigma_{m}+\sigma_{a} \sin (\omega t)
$$

where $\sigma_{m}$ and $\sigma_{a}$ are respectively the mean and alternating normal stress components and $\omega$ is the angular frequency

$$
\begin{gathered}
P_{S W T_{\text {max }}}=\frac{\sigma_{a}\left(\sigma_{\max }\right)}{E} \\
P_{F S_{\text {max }}}=\frac{-\sigma_{a}\left(\cos \left(\theta_{\text {crit }}\right) \sin \left(\theta_{\text {crit }}\right)\right)}{G}\left[1+k \frac{\sigma_{\text {max }} \cos ^{2}\left(\theta_{\text {crit }}\right)}{S_{y}}\right] \\
\theta_{\text {crit }}= \pm \cos ^{-1}\left[\sqrt{\left.\frac{\sqrt{4 S_{y}\left(S_{y}+\alpha\left(\sigma_{\text {max }}\right)\right)+9 k^{2}\left(\sigma_{\max }\right)^{2}}+3 k\left(\sigma_{\text {max }}\right)-2 S_{y}}{8 k\left(\sigma_{\max }\right)}\right]}\right]
\end{gathered}
$$

where $\sigma_{\max }=\sigma_{m}+\sigma_{a}, S_{y}$ is the yield strength and $k$ is a material constant $(0 \leq k \leq 1)$.

\subsection{Calibration of the $l$ versus $N_{f}$ relationship by using multiaxial models and Point Method}

Susmel and Taylor [4] reformulated the TCD by extending its application to predict the fatigue life in the medium cycle. This extension was based on the hypothesis that the critical distance, $l$, varies with the fatigue life according to the relation presented in Equation (8). In this sense, fatigue failure is expected to occur if this effective stress exceeds a reference material fatigue strength. Simplified methods can also be formulated by considering averages over an area or a line (Area and Line Methods, respectively) or the stress at a point located at a critical distance, $l$, from the stress raiser (Point Method).

$$
l\left(N_{f}\right)=A_{l} N_{f}^{b_{l}}
$$

where $A_{l}$ e $b_{l}$ are the material constants. The fitting procedures to obtain these constants require two fatigue curves generated by testing plain and sharply notched specimens under fully reversed push-pull.

Once the material parameter behavior is defined as a function of the number of cycles, it will be possible to use a strategy similar to that proposed by Susmel and Taylor [4] for the construction of the curve that correlates the critical distance based on the multiaxial parameter, $l_{P_{*}}$, with the life of notched specimens. In the strategy applied in this work, it is 
necessary to use, besides the $P_{*}$ versus $N_{f}$ curve, a curve representing the fatigue behavior of the material in the presence of the notch, respectively the elements (a) and (b) shown in Figure 1 (note that fatigue curves can be obtained with mean stress equal or different from zero). First, a range of life that is desired to estimate the $l_{P_{*}}$ versus $N_{f}$ curve is required, for example, between $N_{\text {inf }}$ and $N_{\text {sup }}$. Thus, assuming the $N_{i}$ life, $N_{\text {inf }} \leq N_{i} \leq N_{\text {sup }}$, we can calculate from Figure 1(a) the pair $\left(\sigma_{a_{i}}, \sigma_{m_{i}}\right)$, or $\left(\tau_{a_{i}}, \sigma_{m_{i}}\right)$, and from Figure 1(b) the value of $P_{*}$. The next step is to use the $\left(\sigma_{a_{i}}, \sigma_{m_{i}}\right)$ pair to determine the distribution of the elastic stress field and calculate using the methodology described in item 3.2 the value of $P_{*}$ at a distance $\iota$ from the notch root along the bisector line of the notch, $P_{*}(l)$ (see Figures 1(c) and 1(d)). Once the $P_{*}(\iota)$ is determined, a search process is performed to identify the distance from the notch root, $\iota$, where the value of $P_{*}(\iota)$ is equal to $P_{*}\left(N_{i}\right)$. This value represents a point $\left(l_{P_{*}}, N_{f}\right)$ of the $l_{P_{*}}$ versus $N_{f}$ curve. To obtain the other points, the procedure above described must be repeated and, once the pairs $\left(l_{P_{*}}, N_{f}\right)$ are obtained, the curve parameters can be estimated using non-linear regression techniques.

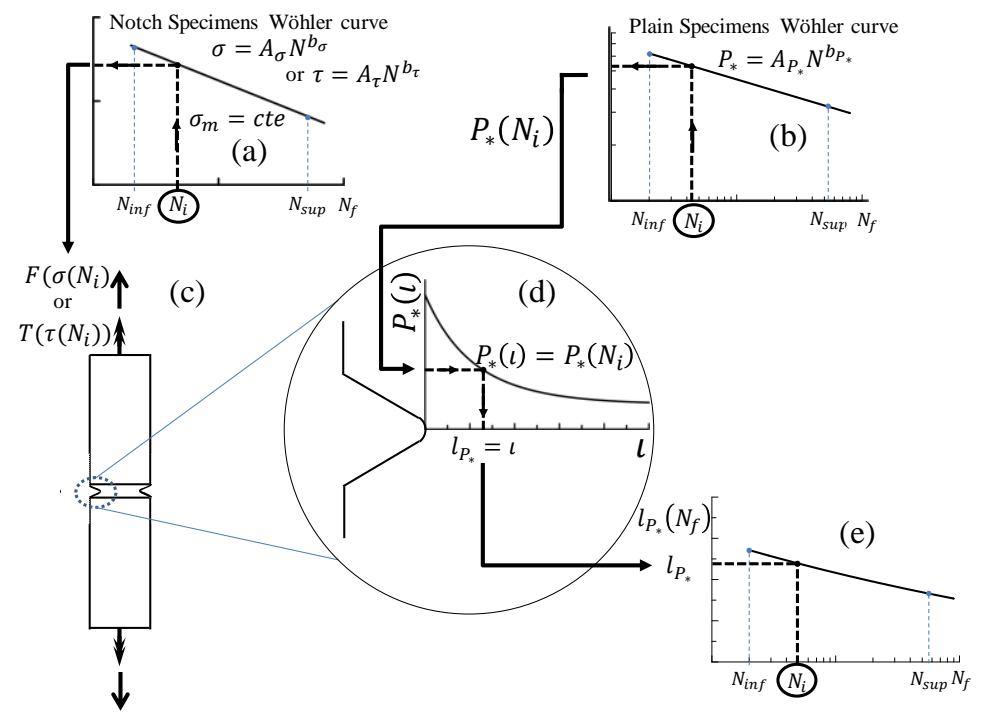

Figure 1 Representation of the basic structure for the characterization of the $\boldsymbol{l}_{\boldsymbol{P}_{*}}$ versus $\boldsymbol{N}_{\boldsymbol{f}}$ curve using the point method

\subsection{Procedure to estimate the fatigue life}

The life estimation was performed by using an iterative process based on bisection method. This algorithm was developed in a Matlab code to compare two fatigue lives: an initial arbitrary value and a material fatigue life obtained from the $P_{*}-N_{f}$ curve representative of the material fatigue behaviour. The initial arbitrary fatigue life is used to obtain the critical distance. From this critical distance, multiaxial parameter $P_{F S}, P_{S W T}$ and $\sigma_{1_{a}}$ is determinate from the linear-elastic distribution along the notch bisector line. The calculated value of the $P_{*}$ parameter allows to estimate the "first failure life" from the $P_{*}-$ $N_{f}$ relation. Now, the two fatigue lives are compared with the objective of identifying the absolute value of the difference between the lives and their signal. The iterative process was stopped when the percentage difference between lives is less than the specified tolerance (tipically $<5 \%$ ). 


\section{Comparison with experimental data}

\subsection{Experimental Data}

We collected fatigue data from smooth and notched specimens made of EN3B steel subjected to axial loading conditions presented in Susmel and Taylor [4]. Tables 1 and 2 show, respectively, the mechanical properties and dimensions of the specimens used for the characterization of the effect of the presence of notches and the stress ratio, $R$, on the fatigue behavior. The geometries of the specimens are shown in Figure 2. In order to process the fatigue data reported in Tables 1 and 2, the graphs presented in Susmel and Taylor [2007] were scanned and processed electronically by the WebPlotDigitizer application [23]. The Wohler parameters reported in Table 2 were estimated in terms of the amplitude of the gross nominal stress.

Table 1 - Mechanical Properties

\begin{tabular}{|c|c|c|c|c|c|c|}
\hline \multirow{2}{*}{$\sigma_{y}[\mathrm{MPa}]$} & \multirow{2}{*}{$\sigma_{u}[\mathrm{MPa}]$} & \multirow{2}{*}{$E[\mathrm{MPa}]$} & \multirow{2}{*}{$\mathrm{R}$} & \multirow{2}{*}{$\begin{array}{c}\text { Number of } \\
\text { Data }\end{array}$} & \multicolumn{2}{|c|}{ Wohler Parameters } \\
\hline & & & & & $\mathrm{A}[\mathrm{MPa}]$ & $\mathrm{b}$ \\
\hline \multirow{2}{*}{606,2} & \multirow{2}{*}{638,5} & \multirow{2}{*}{194700} & -1 & 11 & 853,9 & $-0,0991$ \\
\hline & & & 0,1 & 17 & 1471,0 & $-0,1565$ \\
\hline
\end{tabular}

Table 2 - Dimensions and fatigue properties specimens (dimensions in $\mathrm{mm}$, angles in degree)

\begin{tabular}{|c|c|c|c|c|c|c|c|c|c|c|}
\hline \multirow{2}{*}{$\begin{array}{l}\text { Specimen } \\
\text { Shape }\end{array}$} & \multirow{2}{*}{$W_{g}$} & \multirow{2}{*}{$t$} & \multirow{2}{*}{$r$} & \multirow{2}{*}{$\theta$} & \multirow{2}{*}{$d$} & \multirow{2}{*}{$K_{t_{g}}$} & \multirow{2}{*}{$\mathrm{R}$} & \multirow{2}{*}{$\begin{array}{c}\text { Number } \\
\text { of Data }\end{array}$} & \multicolumn{2}{|c|}{ Wohler Parameters } \\
\hline & & & & & & & & & $A[\mathrm{MPa}]$ & $b$ \\
\hline \multirow[t]{2}{*}{ Fig. 2(a) } & \multirow[t]{2}{*}{25} & \multirow[t]{2}{*}{4} & \multirow[t]{2}{*}{0,12} & \multirow[t]{2}{*}{60} & \multirow[t]{2}{*}{--} & \multirow[t]{2}{*}{15,95} & -1 & 9 & 2138,3 & $-0,2611$ \\
\hline & & & & & & & 0,1 & 17 & 1280,2 & $-0,2479$ \\
\hline \multirow[t]{4}{*}{ Fig. 2(b) } & \multirow[t]{4}{*}{25} & \multirow[t]{4}{*}{--} & \multirow[t]{4}{*}{--} & \multirow[t]{4}{*}{--} & \multirow[t]{2}{*}{8,0} & \multirow[t]{2}{*}{3,44} & -1 & 8 & 740,4 & $-0,1501$ \\
\hline & & & & & & & 0,1 & 8 & 809,9 & $-0,1751$ \\
\hline & & & & & \multirow[t]{2}{*}{3,5} & \multirow[t]{2}{*}{3,07} & -1 & 8 & 1135,0 & $-0,1623$ \\
\hline & & & & & & & 0,1 & 8 & 1000,4 & $-0,1733$ \\
\hline \multirow[t]{2}{*}{ Fig. 2(c) } & \multirow[t]{2}{*}{25} & \multirow[t]{2}{*}{5} & \multirow[t]{2}{*}{1,5} & \multirow[t]{2}{*}{--} & \multirow[t]{2}{*}{--} & \multirow[t]{2}{*}{6,10} & -1 & 9 & 1275,4 & $-0,2241$ \\
\hline & & & & & & & 0,1 & 7 & 860,2 & $-0,2055$ \\
\hline
\end{tabular}

Figure 3 shows the diagrams correlating the fatigue life, $N_{f}$, with the parameters of Fatemi-Socie, $P_{F S}$, the Smith-Watson-Topper, $P_{S W T}$, and the Maximum Principal Stress Amplitude, $\sigma_{1 a}$. In these figures, the square marks represent the experimental results performed with stress ratio, $R$, equal to -1 , while the circles represent the results obtained with $R=0,1$. The dependent variable, fatigue life $\left(N_{f}\right)$, is represented in the abscissa in logarithmic scale and the independent variable $\left(P_{F S}\right.$ or $\left.P_{S W T}\right)$ is represented in the ordinate, also in logarithmic scale. Considering the aspect of the dispersion diagrams presented in Fig. 3 , to represent the relation between the fatigue parameter and the fatigue lives we will use the power function represented by Equation (9)

$$
P_{*}\left(N_{f}\right)=A_{p} N^{b_{p}}
$$

where $A_{p}$ and $b_{p}$ are the fatigue coefficient and exponent obtained according to each parameter considered in the analysis. The trend curves (represented by the solid lines) and the respective upper and lower bounds of the individual prediction intervals (plotted in dashed lines) are obtained using linear regression models under the hypothesis of a log-normal distribution of the number of cycles to failure for each stress level and assuming a confidence level equal to $95 \%$. The syntheses of the generated results, carried out according to the above statistical procedure, are summarized in Table 3. The analysis of Figure 3(a) shows that the use of the FS parameter, 
estimated for $k=1$, provides a good correlation, independent of stress ratios, $R$ (scatter bands sketched of \pm 3 approximately and coefficient of determination, $r^{2}$, equal to 0,73 ). However, the analysis of Figures 3 (b) and 3(c) shows that the use of the $S W T$ and $\sigma_{1_{a}}$ parameter provides a relatively weak correlation (scatter bands sketched greater than 4 . and $r^{2}$ less than 0,70 ), not allowing to consistently represent the effect of the stress ratio, $R$, on the life $N_{f}$.
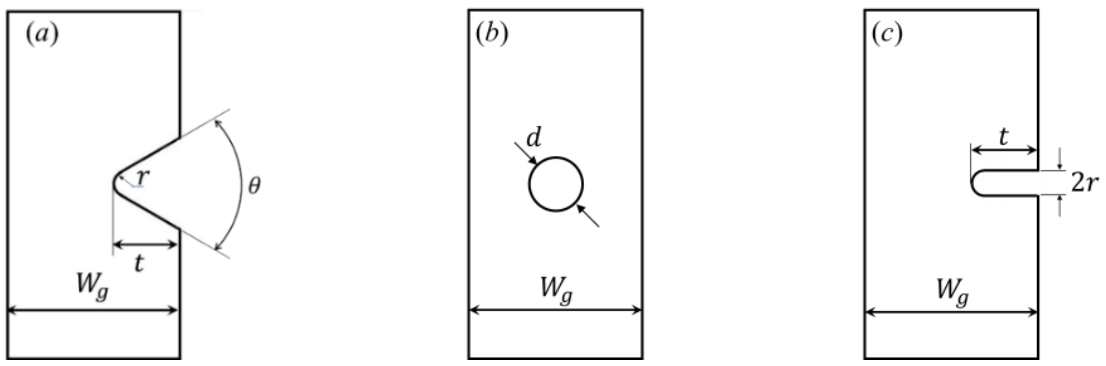

Figure 2 - Specimens geometry. (a) single edge V-notch, (b) hole-type notch, (c) single edge U-notch

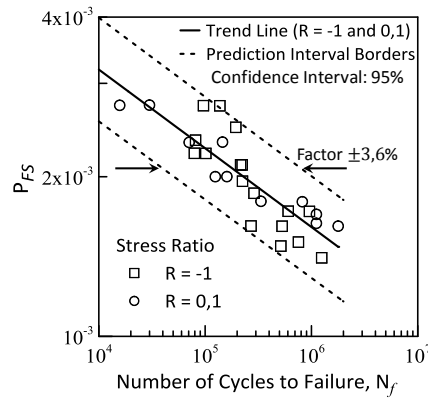

(a)

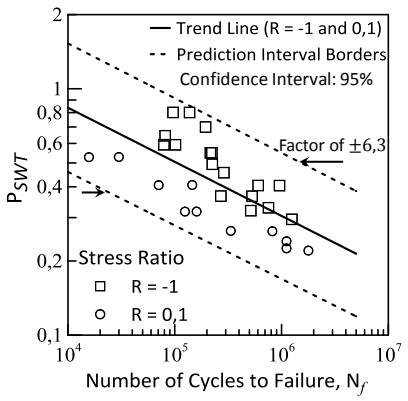

(b)

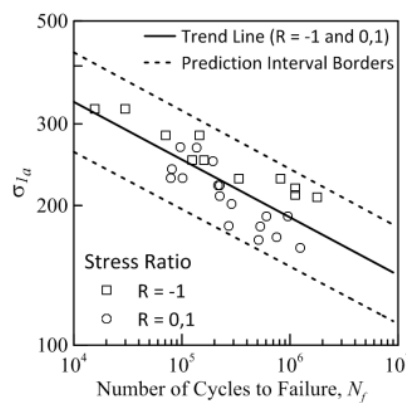

(c)

Figure 3 - Wohler Diagrams - (a) $\boldsymbol{P}_{F S}-\boldsymbol{N}_{f}$; (b) $\boldsymbol{P}_{\boldsymbol{S W T}}-\boldsymbol{N}_{\boldsymbol{f}}$ and (c) $\boldsymbol{\sigma}_{\mathbf{1}_{\boldsymbol{a}}}-\boldsymbol{N}_{\boldsymbol{f}}$.

Table 3 Parameters of Fatigue Curves

\begin{tabular}{c|c|c|c|c}
\hline $\begin{array}{c}\text { Stress Ratio, } \\
R\end{array}$ & Fatigue & Fatigue constant & Fatigue exponent & \multirow{2}{*}{$\mathrm{r}^{2}$} \\
\cline { 3 - 5 } & Model & $A_{p}(\mathrm{MPa})$ & $b_{p}$ & \\
\hline-1 & SWT & 3,694 & $-0,1983$ & 0,92 \\
& FS & $8,743 \mathrm{E}-03$ & $-0,1190$ & 0,92 \\
& $\sigma_{1_{a}}$ & 853,9 & $-0,0992$ & 0,92 \\
\hline 0,1 & $\mathrm{SWT}$ & 24,36 & $-0,3130$ & 0,73 \\
& $\mathrm{FS}$ & $2,577 \mathrm{E}-02$ & $-0,2066$ & 0,73 \\
& $\sigma_{1_{a}}$ & 1471 & $-0,1565$ & 0,73 \\
\hline all data & $\mathrm{SWT}$ & 6,333 & $-0,2196$ & 0,46 \\
& $\mathrm{FS}$ & $1,25 \mathrm{E}-02$ & $-0,1485$ & 0,74 \\
& $\sigma_{1_{a}}$ & 1053 & $-0,1245$ & 0,62 \\
\hline
\end{tabular}

\subsection{Critical distance curves}

The parameters of the $l-N_{f}$ curves presented in Table 4 were obtained considering the application of the calibration procedures described in item 2.2 and 2.3. In this sense, the $l-N_{f}$ curves were estimated by considering the fatigue curves of the $\mathrm{V}$-notches specimens (with stress ratios -1 and 0,1$)$ and the $P-N_{f}$ curves of the plain specimens tested under the same stress ratios. In this table the values of critical distances calculated considering the lower and upper 
limits of life adopted in the calibration process are respectively $10^{4}$ and $10^{6}$ cycles. Analyzing these values, it can be seen that in $10^{6}$ (value typically adopted for the infinite life of steels) the estimates for the critical distance are of the same order of magnitude, independently of the fatigue model and the stress ratio used. However, the estimates of the critical distances performed for $10^{4}$ cycles show a significant effect of the stress ratio, i.e.: for $R=-1, l=1,36$, and for $R=0,1, l=0,48$. Therefore, our results indicate that the $l-N_{f}$ curves show a small dependency on the fatigue models used in the calibration, but apparently a strong dependency of the stress ratio.

Table 4 Parameters of the $l$ versus $N_{f}$ relationships

\begin{tabular}{cccccc}
\hline \hline \multirow{2}{*}{$\begin{array}{c}\text { Stress ratio, } \\
R\end{array}$} & Multiaxial & \multicolumn{2}{c}{ Parameters } & \multicolumn{2}{c}{$l(N)$} \\
\cline { 3 - 6 } & Models & $A_{p}$ & $b_{p}$ & $10^{4}$ & $10^{6}$ \\
\hline \multirow{3}{*}{-1} & SWT & 36,78 & $-0,3578$ & 1,36 & 0,26 \\
& FS & 38,46 & $-0,3618$ & 1,37 & 0,26 \\
& $\sigma_{1_{a}}$ & 36,76 & $-0,3578$ & 1,35 & 0,27 \\
\hline \multirow{2}{*}{0,1} & SWT & 0,5173 & $-0,02331$ & 0,42 & 0,37 \\
& FS & 1,288 & $-0,1064$ & 0,48 & 0,30 \\
& $\sigma_{1_{a}}$ & 2,885 & $-0,1808$ & 0,55 & 0,24 \\
\hline
\end{tabular}

\subsection{Life estimates for the notched specimens}

The life predictions were performed considering the experimental data obtained for the specimens presented in Figure 2. In the life predictions using the FS and SWT models, the results with the two stress ratios were used without distinction. In other words, the calibrated $l-N_{f}$ curve considering the experimental data obtained with $\mathrm{R}=-1$ was used to predict the fatigue life of the tests performed with $R=-1$ and 0,1 . However, in life predictions using the maximum principal stress amplitude, $\sigma_{1_{a}}$, due to the limitations of the parameter, the same strategy used elsewhere [4] was considered, i.e., the predictions obtained considering a set of data tested under a given stress ratio should be performed considering the $\sigma_{1_{a}}-N_{f}$ and $l_{p}-$ $N_{f}$ curves calibrated with identical stress ratios. If this was not the case, there would be variations in life estimates of the order of 4.5 in average terms, reaching a maximum of 35 . The results of the fatigue life estimation are show in Figure 4. These graphs compare the experimental life $\left(N_{f}\right)$ with the estimated one $\left(N_{e s t}\right)$. The straight line represents the perfect fatigue life estimation. The region above this line represents conservative life estimation. The dashed lines represent the factor 3 error bands for the estimated life.

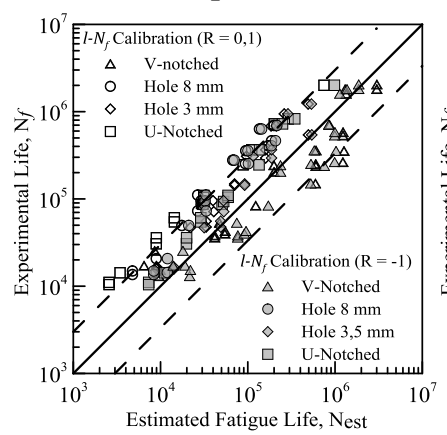

(a)

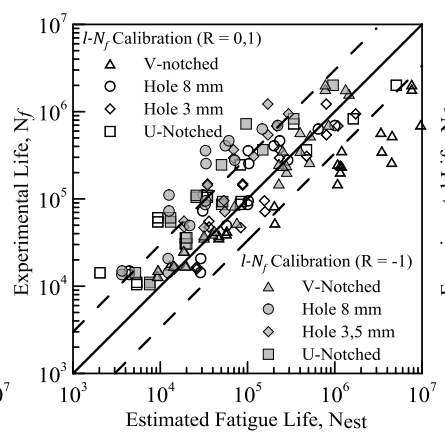

(b)

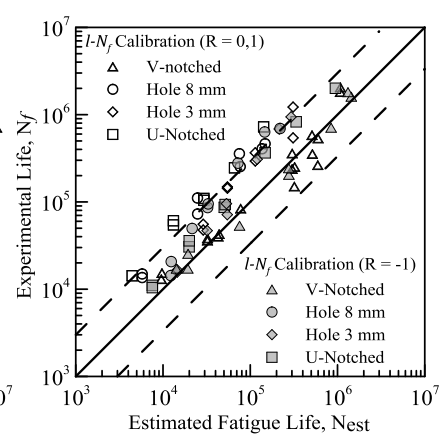

(c)

Figure 4 - Accuracy of the life estimates for the notched specimens - Parameter: (a) Fatemi-Socie ;

(b) Smith-Watson-Topper and (c) maximum principal stress amplitude 
The comparison of these diagrams clearly shows that the life estimates performed using the $l-N_{f}$ curve calibrated from the FS model was the most accurate method, allowing the data points to fall within the parent material scatter band $( \pm 3,6)$, and conservative $(75 \%$ of the estimates ). On the other hand, the predictions made considering the $l-N_{f}$ curve estimated from the SWT model were extremely poor, due to the great dispersion of the results and due to the increase in the number of non-conservative estimates. The authors believe that this behavior is due to the fact that the Fatemi-Socie model represents better the fatigue material behavior.

\section{Conclusions}

In this paper, we demonstrate that multiaxial fatigue models can be used for the calibration of the critical versus life distance curve. In this scenario, we show the procedures necessary for the calibration of the $l-N_{f}$ curve using the point method and the Fatemi-Socie, FS, and SmithWatson-Topper, SWT models. As a consequence, it has been found that, apparently, the $l-N_{f}$ curve is strongly influenced by the stress ratio. The authors claim that this behavior may be associated with statistical variability resulting from the reduced number of experimental data used for the construction of the S-N curves.

\section{References}

1. D. Taylor, Geometrical effects in fatigue: a unifying theoretical model. Int J Fatigue, 21: 413-20 (1999)

2. L. Susmel, D. Taylor, Two methods for predicting the multiaxial fatigue limits of sharp notches, Fatigue Fract Engng Mater Struct, 26: 821-33, (2003).

3. L. Susmel, A unifying approach to estimate the high-cycle fatigue strength of notched components subjected to both uniaxial and multiaxial cyclic loadings, Fatigue Fract Engng Mater Struct, 27: 391-411 (2004).

4. L. Susmel, D. Taylor, A novel formulation of the Theory of Critical Distances to estimate Lifetime of Notched Components on the Medium-Cycle Fatigue Regime, Fatigue Fract Engng Mater Struct, 30: 567-81 (2007)

5. L. Susmel, D Taylor, Can the conventional high-cycle multiaxial fatigue criteria be reinterpreted in terms of the theory of critical distances?. SDHM, 2, 2: 91-108 (2006)

6. T. D. Righiniotis, B. M. Imam, M. K. Chryssanthopoulos, Fatigue analysis of riveted railway bridge connections using the theory of critical distances, Engng Struct, 30, 10 : 2707-15 (2008)

7. M. Benedetti, V. Fontanari, C. Santus, M. Bandini, Notch fatigue behaviour of shot peened high-strength aluminium alloys: Experiments and predictions using a critical distance method, Int J Fatigue, 32, 10: 1600-11 (2010)

8. L. Susmel, D. Taylor, An Elasto-Plastic Reformulation of the Theory of Critical Distances to Estimate Lifetime of Notched Components Failing in the Low/MediumCycle Fatigue Regime, ASME. J. Eng. Mater. Technol., 132, 2 (2010)

9. L. Susmel., Modified Wöhler curve method, theory of critical distances and Eurocode 3: A novel engineering procedure to predict the lifetime of steel welded joints subjected to both uniaxial and multiaxial fatigue loading, Int. J Fatigue, 30, 5: 888-907, (2008)

10. L. Susmel., The theory of critical distances: a review of its applications in fatigue, Eng Fract Mech, 75, 7: 1706-24 (2008)

11. L. Susmel, D. Taylor, The Modified Wöhler Curve Method applied along with the 
Theory of Critical Distances to estimate finite life of notched components subjected to complex multiaxial loading paths, Fatigue Fract Engng Mater Struct, 31, 12: 1047-64 (2008)

12. Y. Yamashita, Y. Ueda, H. Kuroki, M. Shinozaki, Fatigue life prediction of small notched Ti-6Al-4V specimens using critical distance, Engng Fract Mech, 77, 9, 1439 1453, (2010)

13. S. Capetta, R. Tovo, D. Taylor, P. Livieri, Numerical evaluation of fatigue strength on mechanical notched components under multiaxial loadings, Int. J Fatigue, 33, 5, 661-71, (2011)

14. J.F. Flavenot, N. Skalli, A critical depth criterion for the evaluation of long-life fatigue strength under multiaxial loading and a stress gradient, Biaxial and Multiaxial Fatigue, EGF3, p. 355-65 (1989)

15. F.C. Castro, J.A. Araújo, N. Zouain, On the application of multiaxial high-cycle fatigue criteria using the theory of critical distances, Eng Fract Mech, 76, 4: 512-24, (2009).

16. D. Socie., Multiaxial Fatigue Damage Models, J. Eng. Mater. Technol., 109, 4, 293-98 (1987).

17. R. N. Smith, P. Watson, T.H. Topper, A stress-strain parameter for the fatigue of metals, J. Mater, 5, 4: 767-78, (1970)

18. Fatemi A.; Socie D., A critical plane approach to multiaxial fatigue damage including out-of-phase loading, Fatigue Fract Engng Mater Struct, 11, 3, 149-65, (1988)

19. M. W. Brown, K. J. Miller., A theory for fatigue failure under multiaxial stress-strain conditions, Proc. Inst. Mech. Eng., 187, 1: 745-55, (1973)

20. , K. Dang Vang, Sur la résistance à la fatigue des mètaux, Sciences et Techniques de l'Armement, Mémorial de l'Artillerie Française, 1973, p. 3.

21. L. Susmel, A simple and efficient numerical algorithm to determine the orientation of the critical plane in multiaxial fatigue problems, Int. J. Fatigue, 32, 11, 1875-1883, (2010)

22. E. N. Mamiya, J. A. Araujo, Fatigue limit under multiaxial loadings: on the definition of the equivalent shear stress, Mech Res Commun,29, 141-51 (2002)

23. A. Rohatgi, http://arohatgi.info/WebPlotDigitizer/app/(2018). 